\title{
DESIGN AND IMPLEMENTATION OF POWER EFFICIENT SYSTEM USING IOT BASED WIRELESS SENSOR FOR ENVIRONMENT MONITORING
}

\section{U. L. BOMBALE \& SANJIVANI TUKARAM IKKE}

Department of Electronics Technology, Shivaji University, Kolhapur, Maharashtra, India

\begin{abstract}
The rapid growth in industrial processing and fuel fired automobiles are the main cause of environmental pollution. To have control over these entities, automatic pollutants, such as toxic gases are required to be monitored and quantified rapid growth of IOT devices. This paper proposed a development of the automatic monitoring device. This monitoring system measures the levels of temperature, toxic gas, $\mathrm{pH}$, air pressure. For this, an embedded system is developed along with Wi-Fi web server which is capable to display the parameter on HTML pages and also record periodic levels in database software.
\end{abstract}

KEYWORDS: Environment Hazardous Parameters, Environment Sensors \& Internet of Things (IoT)

Received: Mar 19, 2019; Accepted: Apr 09, 2019; Published: Apr 26, 2019; Paper Id.: IJECIERDJUN20193

\section{INTRODUCTION}

The importance of monitoring is existed in many aspects. The weather conditions changes day by day, so to monitor and maintain the healthy growth in crops and to ensure the safe working environment in farming, industries, etc [1] is must. As technology grows, the process of recording the environmental parameters became easier compared to the past days. The most common parameters being monitored includes the temperature, humidity, rainfall, atmospheric pressure, light intensity, air quality affected by pollutants viz. $\mathrm{CO}_{2}, \mathrm{CO}$ and many others [2] [3].

The majority of monitoring applications based on WSNs, due to its advantages such as lower costs due to the replacement of cables, various network topologies, scalability, lower maintenance and commissioning expenses [4]. Wireless sensor networks have been successfully used in various fields such as, environmental monitoring [5] [7], current consumption monitoring in large buildings, healthcare applications, location tracking, hazardous gases and process control in industry, natural disaster prevention, and many others [8] -[14]. The most of the applications used for environmental monitoring, consist of ZigBee (IEEE 802.15.4), Wi-Fi (based on IEEE 802.11), and Bluetooth (IEEE 802.15.1). Which having less compact latency and reliability requirements, such as Wi-Fi technology based on the IEEE 802.11 it offers higher transmission range [15]. 


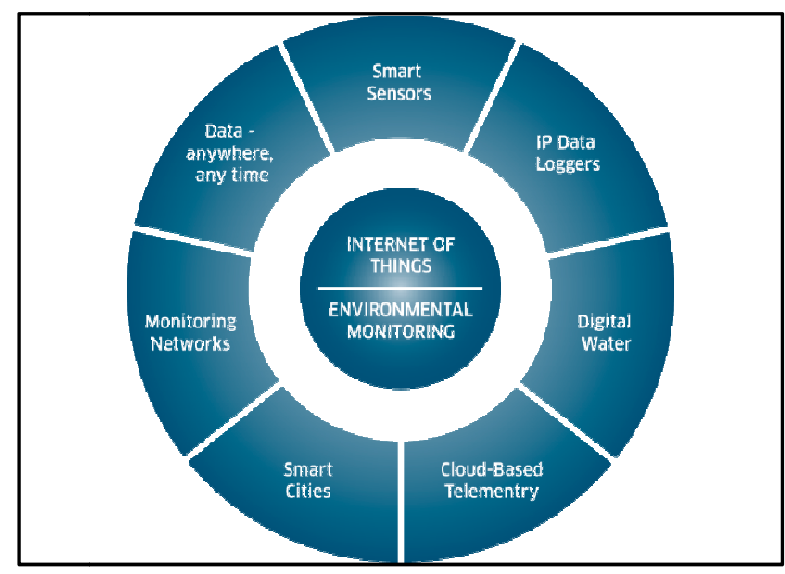

Figure 1: Environment Monitoring

Wi-Fi is a popular networking technology based on the IEEE 802.11 set of standards that offers higher transmission range and throughput compared to IEEE 802.15.4, with the cost of higher energy consumption. By using internet connectivity we can easily measure environmental parameter for sending and receiving measure data.

For transmission and execution of huge amount of data regarding to observe environmental parameters, new system is created which called Internet of Things (IoT). The present work based on IoT wireless sensors which employs User Datagram Protocol (UDP)-based communicating through Wi-Fi and Hypertext Transfer Protocol (HTTP) by using Wi-Fi modules for environmental monitoring.

\section{SYSTEM OVERVIEW}

Figure 2 shows the functional block diagram of the system. The system has been designed in such a way that it takes several inputs i.e. sensors, communication Module, user interface, battery ect. The inputs from the sensors are integrated and processed with controller named Arduino. The results are sent through the communication module to standstill and monitoring system. The results are displayed on HTML based web server and record data to MySQL server(excel sheet) that displays status of multi time variation of environmental parameter on PC using login provided. Also for efficient power 12v 7A rechargeable battery is used. Therefore, the processor in the developed devices is in charge with performing all the actions for the proper operation of the device, namely, power management, acquisition of data from the sensing unit, and communication. For transmitting the data to a base station, a serial link between the processor and the communication module ESP8266. The motivation for selecting the ESP8266 wireless module consists in its ultra-lowpower operation, it consumes about than 60uA in deep sleep mode (with RTC clock still running) and less than 1.0mA or less than $0.5 \mathrm{~mA}$ to stay connected to the access point. The design is modular as a result it is easy and straight forward to add extra sensors for measuring and monitoring environmental parameters. The sensing systems are explained in details in the further section. 


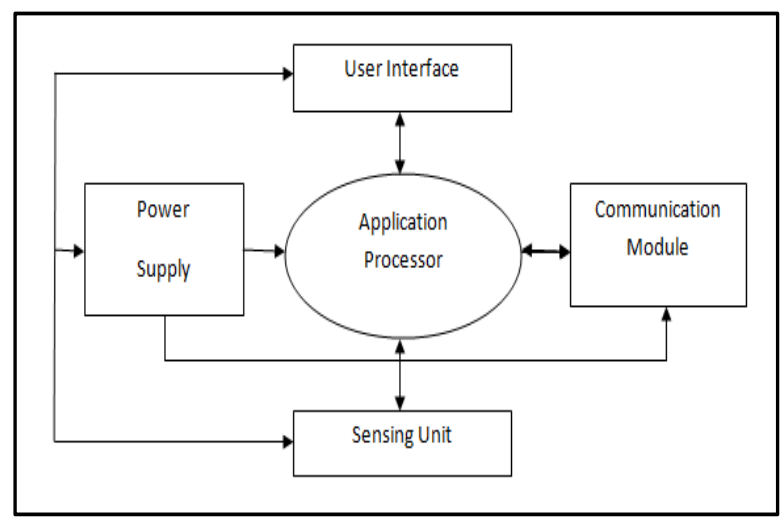

Figure 2: Block Diagram of Hardware System

\section{DETAILS OF SYSTEM}

The component used in the system is explained in details are as follows,

\section{Temperature and Humidity Sensor}

DHT11 Temperature \& Humidity Sensor has complex sensor with a calibrated digital signal output. It gives high reliability and excellent long-term stability. This sensor contains resistive-type component for humidity measurement and an NTC component for temperature measurement. DHT11 having excellent quality, fast response, anti-interference ability and cost-effectiveness.

\section{Gas Sensor}

Depending on the sensitivity levels various specifications of Gas sensors are available, type of gas to be sensed. When a gas interacts with sensor, it is first ionized into constituents and is then adsorbed by the sensing element. This adsorption generates a potential difference on the element which is send to the processor unit through output pins in form of current.

\section{- MQ4 Sensor}

The MQ-4 gas sensor is highly sensitive to SnO2, which has lower conductivity of clean air. When the target combustible gas exists, the sensor's conductivity gets higher with the gas concentration increases. Then we can analyse the change of conductivity to correspond output signal of gas concentration through a simple circuit. The MQ-4 gas sensor has a higher sensitivity to methane, also has anti-interference to alcohol and other gases.

\section{- $\quad$ MQ6}

The MQ6 is a use for liquefied petroleum gas (LPG) sensor. It can be used in gas leakage detecting equipment in consumer and industry applications, this sensor is suitable for detecting LPG, iso-butane, propane, LNG. The sensitivity can be adjusted by the potentiometer.

\section{Air Pressure}

The BMP180 contain a piezo-resistive sensor, an ADC converter and a control unit with E2PROM and a serial I2C interface. The working principle of atmospheric pressure sensor based on the air weight. When the air weight is changed then resistance of piezo resistive sensor is changed. Because this changes in value of analog value this value is 
converted to digital value through analogue to digital converter. Then this value is control through control unit and eventually it is sent to controller using $12 \mathrm{C}$ serial interface. By using BMP180 we can also measure the temperature in ${ }^{\circ} \mathrm{C}$ and pressure in $\mathrm{hPa}$.

\section{pH Sensor}

In dry soil conductivity is very slow, part of soil structure when made dump or moist conductivity increases, these conductivity depends on various parameter few of them are listed as:

- The conductivity of water used

- Contends of soils

- $\quad \mathrm{pH}$ level of both water and soli

Out of these three when $1^{\text {st }}$ two are kept constant variation in conductivity solely depends on $\mathrm{pH}$ level of the contents. It is possible to obtain ph level $\mathrm{b}$ trial and experimentation methods in lab.

\section{SYSTEM IMPLEMENTATION}

The proposed work for Design and implement power efficient system for environment monitoring using IoT based wireless sensor the design details, the development, and different sensors that enable the achieving of Internet connected for monitoring the environment at various locations: one employing UDP-based Wi-Fi communication, one based on the HTTP protocol, and one consisting in power harvesting with efficient power supply. Being provided with Internet connection capabilities, the developed sensors represent a part of the Internet of Things (IoT).

So that present work is divided into following part-

\section{UDP}

Figure 3 presents an UDP-based cyber-physical system For monitoring the temperature and relative humidity, gas sensor, air pressure. Environmental monitoring system with communication based on UDP. This takes advantage of the existing IEEE 802.11 infrastructure for sending measurement information to a cloud-based server platform and provides the possibility of visualizing the data from every device with an Internet connection. Using UDP allows the low-power operation of the Wi-Fi sensors, because of its connectionless nature. Furthermore, this protocol provides lower packet sizes, increased speeds and low latency.

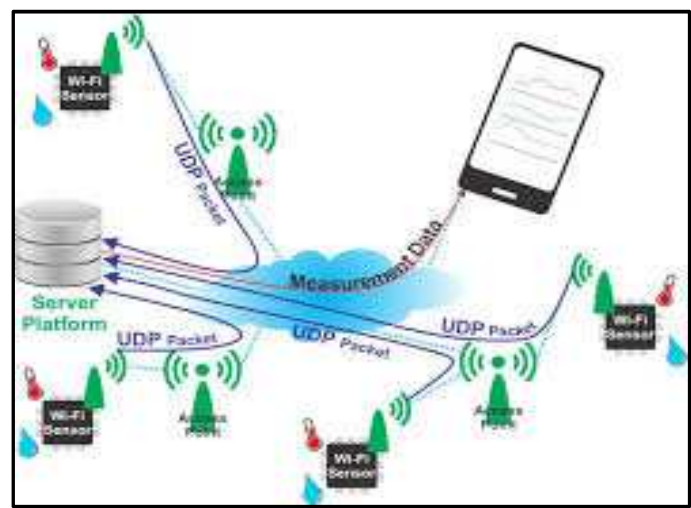

Figure 3: Environmental Monitoring System with Communication Based on UDP 


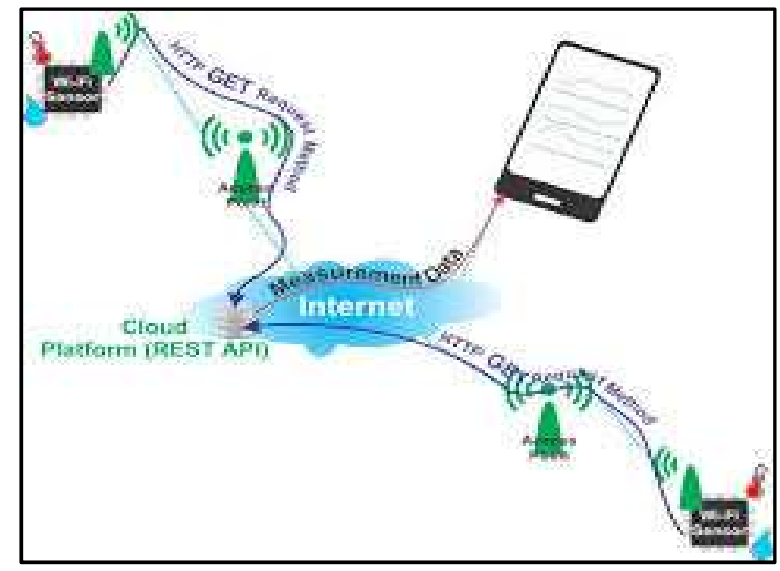

Figure 4: Environmental Monitoring System with Communication Based on HTTP

By using Wi-Fi and UDP lead to the development of an HTTP-based solution, based on the same hardware as the one presented in Figures 2. Therefore, sensors that connect to a WLAN, that send measurement data through HTTP requests. The operation of the wireless sensor is similar to UDP, the only difference is in the way in which data are transmitted. Here, during the wakeup period, the device examines the attached sensor, opens an HTTP connection, and sends the data in the form of a GET request. After receiving the response from the server, it closes the connection and goes to sleep, according to user set the period. For testing the device operation, a generic free public cloud service was selected, data.sparkfun.com, which provides the possibility of logging data from remote locations, according to the IoT vision. The data are posted here through the use of GET and POST requests, based on a public key and on a private key provided by the cloud platform when creating a data stream. These can be accessed from everywhere and the related information can be retrieved in different formats.

\section{Wifi Module Esp8266}

ESP8266 module has better memory capacity and hence web server implementation is feasible. In our system WiFi module receive sensor dada serially from controller. It is provided with interface to database software through SQL programming hence it can record data on PC connected on same WiFi network and bound to fixed IP address.

\section{Power Efficient System}

The applications running on the developed wireless environmental sensors have duty-cycled systems that spend most of the time in sleep mode, and wakeup periodically for taking measurements and sending the data to the server. All sensor and wifi technology consume large power also UDP based communication leads to a larger number of lost packets and satisfactory power consumption. By using HTTP requests, the transmission success ratio increased, but only with a small percentage with the cost of a significant decrease in battery lifetime. Hence power consumption can be calculate as follow

Power consume per sensor $=5 \mathrm{v} * 20 \mathrm{ma}$

$$
=10 \mathrm{~mW}
$$

Power consumed by serial communication per pin for transmitter and receiver $10 \mathrm{~mA}$ 
Average power $=20 \mathrm{~mW}$

Power consumed $\mathrm{b}$ WiFi module $=80 \mathrm{~mW}$

Then total power consumed by all sensor are

Power $=50 \mathrm{~mW}+20 \mathrm{~mW}+80 \mathrm{mw}$

$=150 \mathrm{~mW}$

Energy in terms of time $=1 \mathrm{~s} * 150 \mathrm{~mW}$

$$
=150 \mathrm{mWs}
$$

Then,

Total time for rechargeable battery is $=85000 / 150$

$$
=567 \text { hours }
$$

One full battery can provide $20+$ das backup and again battery can charge by solar panel, so system is energy efficient.

\section{CONCLUSIONS}

The proposed system developed simple power efficient environment monitoring system to measure levels of temperature, toxic gas, $\mathrm{pH}$, air pressure. The developed system embedded with WiFi web server which is capable to display this parameter on HTML pages and also record periodic levels in MYSQL database software.

\section{REFERENCES}

1. R. K. Pachauri and L. A. Meyer, Eds., Climate Change 2014: SynthesisReport. Contribution of Working Groups I, II and III to the FifthAssessment Report of the Intergovernmental Panel on Climate ChangelCore Writing Team, IPCC, Geneva, Switzerland, 2014, p. 151

2. S. Mukhopadhyay, "Research activities on sensing, instrumentation, and measurement: New zealand perspective," IEEE Instrum. Meas. Mag.,vol. 19, no. 2, pp. 32-38, Apr. 2016.

3. J. Gutierrez, J. F. Villa-Medina, A. Nieto-Garibay, andM. A. Porta-Gandara, "Automated irrigation system using a wirelesssensor network and GPRS module," IEEE Trans. Instrum. Meas.,vol. 63, no. 1, pp. 166-176, Jan. 2014.

4. N. Harris, A. Cranny, M. Rivers, K. Smettem, and E. G. Barrett-Lennard, "Application of distributed wireless chloride sensors to environmentalmonitoring: Initial results,” IEEE Trans. Instrum. Meas., vol. 65, no. 4,pp. 736-743, Apr. 2016.

5. [5] M. T. Lazarescu, "Design of a WSN platform for long-term environmental monitoring for IoT applications," IEEE J. Emerg. Sel. Topics CircuitsSyst., vol. 3, no. 1, pp. 45-54, Mar. 2013.

6. O. Postolache, J. D. Pereira, and P. S. Giraİfo, "Wireless sensor networkbased solution for environmental monitoring: Water quality assessment case study,” IET Sci., Meas. Technol., vol. 8, no. 6, pp. 610-616, 2014.

7. Y. Liu, Y. He, M. Li, J. Wang, K. Liu, and X. Li, "Does wireless sensor network scale? A measurement study on GreenOrbs," IEEE Trans.Parallel Distrib. Syst., vol. 24, no. 10, pp. 1983-1993, Oct. 2013.

8. G. Werner-Allen et al., "Deploying a wireless sensor network on an active volcano," IEEE Internet Comput., vol. 10, no. 2, pp. 18-25, Mar. 2006. 
9. J. P. Amaro, R. Cortesão, J. Landeck, and F. J. T. E. Ferreira, "Harvested power wireless sensor network solution for disaggregated current estimation in large buildings,” IEEE Trans. Instrum. Meas., vol. 64, no. 7, pp. 1847-1857, Jul. 2015.

10. Sharma, U., \& Kumar, R. A. J. E. S. H. (2014). Modeling of smart capacitive humidity sensor using ANN. International Journal of Research in Engineering \& Technology, 2, 265-272.

11. D. Magalotti, P. Placidi, M. Paolucci, A. Scorzoni, and L. Servoli, "Experimental characterization of a wireless personal sensor node for the dosimetry during interventional radiology procedures," IEEE Trans.Instrum. Meas., vol. 65, no. 5, pp. 1070-1078, May 2016.

12. D. Magalotti, P. Placidi, M. Dionigi, A. Scorzoni, and L. Servoli, "Experimental characterization of a personal wireless sensor network for the medical X-ray dosimetry,” IEEE Trans. Instrum. Meas., vol. 65, no. 9, pp. 2002-2011, Sep. 2016.

13. M. C. R. Talampas and K. S. Low, "An enhanced geometric filter algorithm with channel diversity for device-free localization," IEEETrans. Instrum. Meas., vol. 65, no. 2, pp. 378-387, Feb. 2016.

14. M. L. Cao, Q. H. Meng, Y. Q. Jing, J. Y. Wang, and M. Zeng, "Distributed sequential location estimation of a gas source via convex combination in wsns," IEEE Trans. Instrum. Meas., vol. 65, no. 6, pp. 1484-1494, Jun. 2016.

15. M. D. Prieto, D. Z. Millan, W.Wang, A. M. Ortiz, J. A. O. Redondo, and L. R. Martinez, "Self-powered wireless sensor applied to gear diagnosis based on acoustic emission," IEEE Trans. Instrum. Meas., vol. 65, no. 1, pp. 15-24, Jan. 2016.

16. Sarmah, K., \& Bhattacharjee, U. (2015). Text-Independent Multi-Sensor Speaker Verification System.

17. G. Mois, T. Sanislav, and S. C. Folea, “A cyber-physical system for environmental monitoring," IEEE Trans. Instrum. Meas., vol. 65, no. 6, pp. 1463-1471, Jun. 2016. 
